\title{
THE DISTRIBUTION OF 35S-LABELLED ETISUL IN THE SKIN AS INDICATED BY AUTORADIOGRAPHY
}

\author{
by D. G. Jamison and Elisabeth Palmer \\ (Department of Human Anatomy, Oxford)
}

The treatment of tuberculosis with "Etisul" (diethyl dithiol isophthalate) was shown by Davies and Driver (1956/7) to be highly successful and this suggested that it might also be useful in the treatment of leprosy. The initial clinical trials in cases with leprosy were carried out by Davey and Hogerzeil (1959) by inunction through the skin and they found the drug to be a useful chemo-therapeutic agent. Although it has not proved to be universally successful in the treatment of all cases and has certain drawbacks, there is no doubt that it has a place in the treatment of leprosy at the moment. For this reason it was considered worth while to determine whether one or more of the tissue components in the skin had a particular affinity for the drug. The work of Snow (1959) has established that 35Slabelled diethyl disulphide can be detected in all body tissues by the assay method after either subcutaneous injection or oral administration in mice and guinea pigs. To map the distribution of "Etisul" in human skin a 35S-labelled preparation was used in the treatment of four patients with leprosy and skin biopsies taken at various intervals following its inunction. These were sectioned in a plane vertical to the surface and the position of the radio-active molecules determined in relation to the various skin layers (and the tissue elements within them) by autoradiography.

\section{Material}

Four cases of leprosy with commonly encountered forms of the disease were selected from among the leprosy patients of the Sudan Interior Mission leprosy settlement in Katsina, Northern Nigeria. Three of the cases were under treatment with dapsone and one with sulphetrone and all appeared to be responding more or less satisfactorily to treatment as judged clinically. None had as yet been treated with "Etisul".

Case 1. (No. 130)

A male aged 30 who was admitted with dimorphous leprosy in 1956 and, despite treatment, in 1959 his ulnar had become sufficiently involved to require neurolysis.

Case 2. (No. 131)

A male aged 38 who was admitted with multiple dimorphous patches in 1960 . 
Case 3. (No. 132)

A male aged 25 who was admitted 3 months previously with a single hypo-pigmented patch over his face which had almost completely disappeared at the time of examination.

Case 4. (No. 133)

A male aged 27 who was admitted in 1955 with lepromatous leprosy. His response to treatment was low and he went into "reaction" as soon as the dose of dapsone was increased sufficiently to become effective. For this reason sulphetrone therapy had to be substituted in 1959.

\section{Method of Treatment with "Etisul"}

The skin covering the arm and forearm on one side of the body was scrubbed for 10 minutes with a soft nailbrush in soap and water. It was then examined clinically. Skin lesions of any kind were recorded and the state of the ulnar, median and radial nerves was determined by palpation. A suitable area was chosen for taking biopsies and the sensory activity of the skin carefully determined. $4.6 \mathrm{ml}$ of "Etisul" containing $50 \mu \mathrm{C}$ of $35 \mathrm{~S}$ was then brushed over the shoulder, the extensor surfaces of the arm, forearm and hand. The concentration of $35 \mathrm{~S}$ was well within the limits of safety laid down by the International Commission for Radiological Protection, according to which $100 \mu \mathrm{C}$ of $35 \mathrm{~S}$ is the maximum permissible dose, assuming that $10 \%$ of the initial dose will be concentrated in the skin.

3 four-millimeter punch biposies were taken from each patient at 3,6 , and 24 hours after the application and fixed in neutral formalin for 13 days. Each specimen was then cut in half: one half being embedded in wax and cut into sections which were stained either with haematoxylin and eosin or with carbol-fuchsin by the FiteFaraco method. The other half was cut on a freezing microtome into a series of 50 sections which were mounted serially, alternate sections being prepared for autoradiography and silver-staining for nerve fibres. The sections destined for autoradiography were briefly washed in distilled water and transferred to microscope slides on which there was a thin layer of gelatine and allowed to dry. They were then covered with Kodak autoradiographic Stripping, AR.10, and left in the dark for periods of 1, 2, 6 and 8 weeks, then developed, fixed and stained with either haematoxylin or toluidin blue.

\section{Observations}

For the purpose of this investigation skin was removed from a selected (standard) area on the back of the forearm in every patient so that the basic histological and neurological patterns would be as comparable as possible from one case to another. 


\section{Plate J}

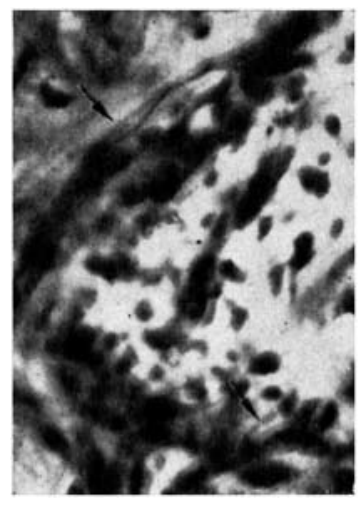

FIG. 1

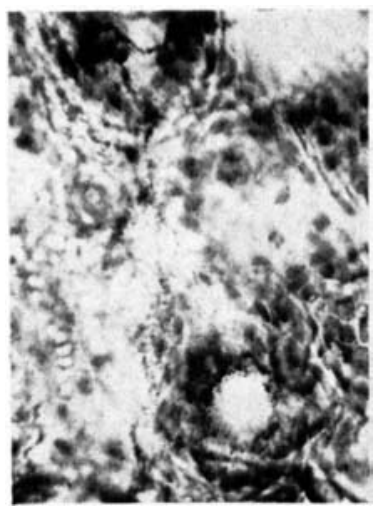

Fig. 3

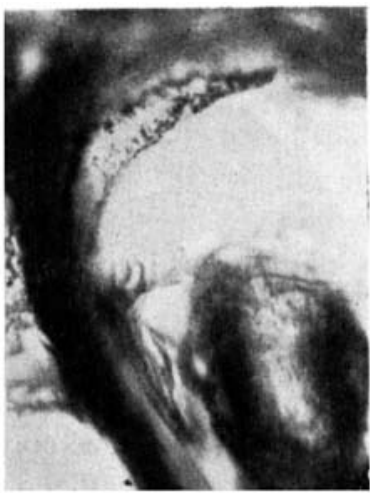

FIG. 2

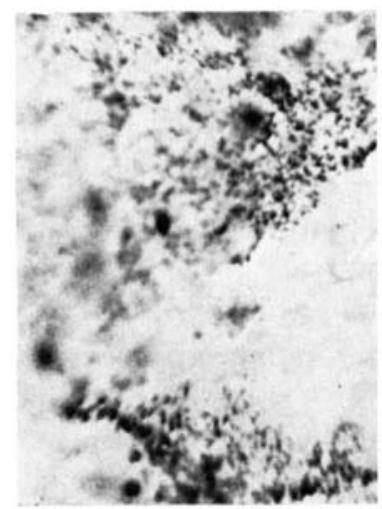

FIG. 4

Fig. 1 Case 1 (130) A single nerve fibre accompanied by large Schwann nuclei in an enlarged sheath of Schwann. $50 \mu$ section, stained Bielschowsky Schofield.

x 550

Fig. 2 Case 1 (130) Autoradiograph of a hairshaft, cut slightly obliquely, showing an accumulation of silver particles between the shaft and the external root sheath in a section from a biopsy 3 hours after treatment.

x 550

FIG. 3 Case 2 (131) Autoradiographs of the sweat gland ducts, cut transversely, the lumen and some of the cells outlined by silver particles. Some silver particles are also outlining cells in the connective tissue around the ducts. Section from a biopsy taken 24 hours after treatment.

x 550

FIg. 4 Case 3 (132) Autoradiograph from a skin biopsy taken 3 hours after treatment. Silver particles are concentrated over the outer root sheath and are also seen outlining individual cells. 


\section{Case 1}

At the time of examination the patient appeared in good health and had no signs of "reaction" to the dapsone he was taking. Following the stripping of his ulnar nerve 9 months previously there had been a steady improvement in the strength of his hand and at the time of examination only slight weakness and wasting of the ulnar muscles was observed. However, sensory acuity was considerably diminished over the dorsal surface of his forearm. The biopsy was taken from the area already selected as standard. This removed skin showed no outward signs of disease whatever. In sections stained with haematoxylin/eosin however there was a sub-epidermal cellular infiltrate and the neuro-vascular bundles were heavily infiltrated with cells, as were the skin structures related to skin appendages. The infiltrate was composed of epithelioid cells, plasma cells and lymphocytes. In some portions of the neurovascular bundles there was connective tissue proliferation and aggregations of fibroblasts. In the section stained with carbolfuchsin there were no acid-fast bacilli to be seen. In the sections stained with silver many regenerating nerve fibres were to be seen but relatively few degenerating ones. The Schwann sheath and the Schwann nuclei were enlarged (Fig. 1).

The autoradiographs made from sections of the biopsy taken three hours after application of 35S-labelled Etisul revealed that radio-active particles had been concentrated between the hair shaft and the external root sheath (Fig. 2) and in mast cells accompanying the superficial blood vessels just beneath the epidermis. In section from biopsies taken 6 hours after application the radio-active particles had concentrated in the cells composing the sub-epidermal infiltrate and also in the cells forming the coils and ducts of the sweat glands. In sections taken 24 hours after application the radio-active particles were again seen concentrated in the cells of the sub-epidermal infiltrate, as well as those surrounding the neuro-vascular bundles. Concentrations of radio-active particles were also seen in the cells composing the outer root sheath of hair follicles and on the whole activity was greater than in the two previous biopsies.

\section{Case 2}

This patient still had a depigmented macule on the extensor surface of his left forearm but the majority of those present on admission were now no longer visible. Sensory testing showed that the area of depigmentation on the back of the left forearm was completely anaesthetic to light touch.

The sections from the biopsy taken from the selected zone of the forearm (which happened to be within the depigmented area) stained with haematoxylin/eosin showed small patches of sub-epidermal infiltrate as a slight aggregation of cells around the neurovascular 
bundles. The sub-epidermal infiltrate consisted mainly of macrophages containing melanin granules as well as numerous mast cells. No acid-fast bacilli were seen in the sections stained with carbol fuchsin. The sections stained with silver that there were no nerve fibres just beneath the epidermis and in the deeper layer of the dermis there were seen nerve bundles containing only fine axons and many empty Schwann tubes.

The autoradiographs made from sections of the biopsy taken three hours after the application of 35S-labelled Etisul showed that there had been a concentration of radio-active particles throughout the sub-epidermal region which contained the cellular infiltrate and also to a lesser extent in the connective tissue zones which contained no adventitial cells. In the autoradiographs from sections taken 6 hours after treatment, radio-active particles were most concentrated in tissue mast cells in the dermis and in cells forming the sweat gland coils in the deeper layers of the dermis. At 24 hours after treatment radio-active particles were found to be denser in the cells of the subepidermal infiltrate than had been the case in sections from the two previous biopsies, and particles were again concentrated in the cells of the coils and ducts of the sweat glands (Fig. 3).

\section{Case 3}

The patch on the patient's face was only just visible and no other clinical signs of leprosy could be detected. Sensory tests over the back of the right forearm showed a slight but significant degree of misreference when compared with that found in this region in average normal subjects, but there was no anaesthesia to light touch. Sections from the biopsy stained with haematoxylin/eosin showed that there was a small increase in the number of cells surrounding all the neurovascular bundles in the dermis; those stained with silver showed a full complement of nerve fibres but the Schwann cells were larger than normal. Some of the deeper nerve bundles contained a few fine axons which had clearly regenerated and they also contained a number of empty Schwann tubes. No acid-fast bacilli were seen in the carbol-fuchsin stained sections.

The autoradiographs from sections of the biopsy taken 3 hours after treatment showed that there was a concentration of radioactive particles surrounding the shaft of the hair itself, but there was also a concentration around the cells forming the outer root sheath (Fig. 4) and the individual cells were outlined by particles of exposed silver. After 6 hours treatment biopsies showed that radio-activity was almost entirely confined in and around mast cells and other cells seen in the connective tissue forming the dermis. After 24 hours treatment the picture was similar but the concentration of radioactive particles was far denser. In addition, radio-active particles 


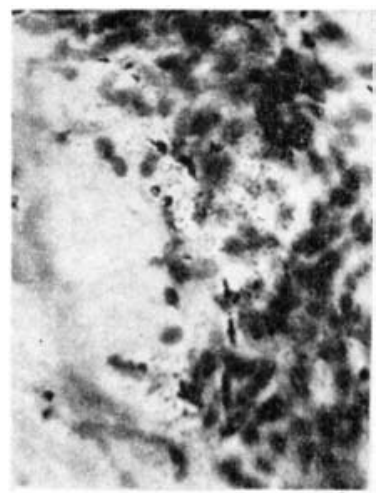

FIG. 5

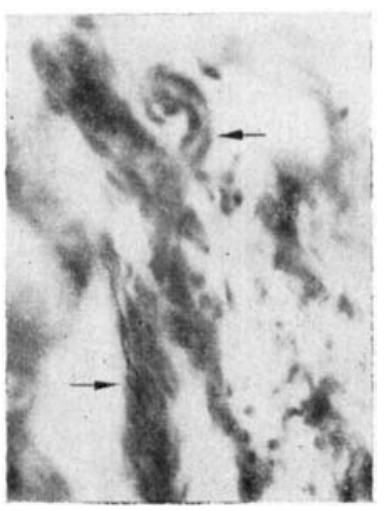

FICi. 7

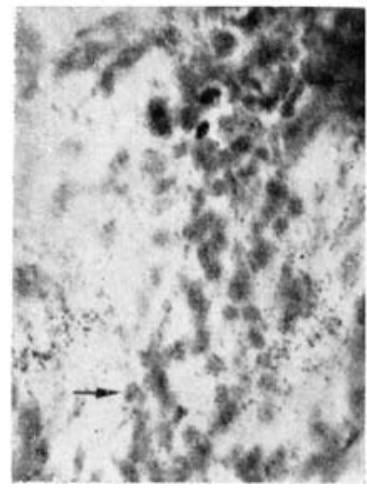

FIG. 6

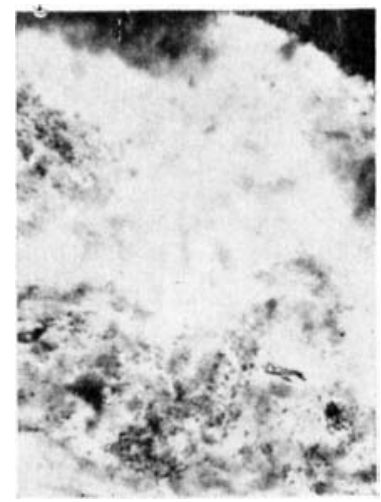

FIG. 8

FIG. 5 Case 3 (132) An autoradiograph from a skin biopsy taken 24 hours after treatment. Silver particles are seen above the cells forming the infiltrate in the dermis.

x 550

FIC. 6 Case 3 (132) Autoradiograph from a skin biopsy taken 24 hours after treatment. The silver particles are concentrated above the nerve bundle and the cells forming the neuro-vascular infiltrate.

x 550

FIG. 7 Case 3 (132) The adjoining section to that shown in Fig. 6. The silverstained preparation shows the same nerve bundle and its position in the infiltrate.

x 550

FIG. 8 Case 4 (133) Autoradiograph from a skin biopsy taken 24 hours after treatment. Silver particles are clearly seen above the lepromatous infiltrate in the sub-epidermis. 
were now seen to be concentrated in large numbers in the ducts leading from the sweat glands and also in the cells forming the infiltrate surrounding these ducts.

\section{Case 4}

On examination, this case showed signs of diffuse lepromatous infiltration and he had no generalized reaction to sulphetrone. Sensory testing over the back of the forearm showed no diminution of sensory acuity.

Sections from the biopsy stained with haematoxylin/eosin showed heavy infiltration of cells around the neuro-vascular bundles and around the tissues of the skin appendages. The infiltrate was particularly dense around the sweat gland cells of the deeper dermis and the infiltrate consisted chiefly of plasma cells, lymphocytes, mast cells and fibrocytes. In some zones of the infiltrate collagen fibres had been laid down. No acid-fast bacilli were seen in the sections stained with carbol-fuchsin. Sections stained with silver showed that the skin was supplied with a normal complement of nerve fibres throughout.

Autoradiographs from biopsies taken 3 hours after treatment showed that there had been little concentration of radio-active particles in the sub-epidermal tissues or in relation to the ducts of the sweat glands. 6 hours after treatment the picture had not changed appreciably, except that the radio-active particles were now more concentrated in the sub-epidermal region. 24 hours after treatment the radio-active particles were seen densely concentrated in and around the cells forming the infiltrate around the neuro-vascular bundles and fewer particles were seen in the cells forming the infiltrate deeper in the dermis (Fig. 5).

In an attempt to follow the distribution of radio-active particles in the skin 48 hours after treatment further biopsies were taken from the standard region on the treated side and a single specimen was also taken from the contra-lateral side of the body. Radio-active particles were present in small numbers in the cells surrounding the neuro-vascular bundles on the untreated side and on the treated side particles were also present in the cells of the sweat gland coils and the cells surrounding the neuro-vascular bundles in the deep dermis.

\section{Discussion}

It is known that the thickness of the epidermis varies greatly from one part of the body to another and it also seems probable that there are slight variations related to sex and age in any given region, but these are not very extensive. Because of this we chose a standard area of skin taken from males between the ages of 25 and 37 years. The site chosen was the extensor surface of the forearm where the 
epidermal thickness is fairly uniform throughout, and the density of innervation is also uniform.

In each case the 35S-labelled Etisul was applied by brush only from the shoulder to the back of the hand and the liquid allowed to penetrate the skin without additional inunction. Care was taken to prevent loss of Etisul by keeping the patients out of the sun to prevent sweating and keeping the arm completely at rest in a horizontal position for 20 minutes following the application of the drug. At the end of this time the liquid had disappeared from the surface of the skin.

In Fig. 2 which is from a section of a biopsy taken three hours after the application of the drug, there is a large concentration of "exposed" silver particles between the hair shaft and the external root sheath close to the epidermis. In other sections taken after a comparable time interval following application of the drug small concentration of exposed silver particles were found in the subepidermal region. This suggests that the drug enters the skin directly through the epidermis and possibly by passing down beside the hair shafts emerging through the epidermis.

Six hours after application of the drug the radio-active particles had become more concentrated in mast cells and in the connective tissue elements composing the infiltrate. It appeared that the concentration of radio-activity was always greatest in the regions where there was reason to suppose that cells were actively accumulating or multiplying.

In cases 132 and 133, in which the sub-epidermal nerve plexus was present, it was possible to show that in sections from biopsies taken 24 hours after treatment there was a concentration of "exposed" silver particles in the position occupied by these nerve bundles. Since they were surrounded by adventitial cells it was not possible to determine the relationship between the particles and any particular nerve element (Figs. 6 and 7).

Twenty-four hours after application of the drug radio-active particles were much more concentrated in and around the adventitial cells characteristic of leprosy in the skin (Fig. 8). They had also been in high concentration in the cells and lumen of the sweat gland ducts. This was also the case after 48 hours. These findings suggest that radio-active particles contained in the drug are partly excreted in the sweat, but some must enter the bloodstream to appear on the opposite side of the body after 48 hours. Lechat (1960) found that Etisul was excreted in the sweat for up to two days after its intitial application and this fits in well with our own observations.

The rapid penetration of radio-active sulphur into the skin and its selective localisation in the pathological zones parallels our clinical findings with unlabelled Etisul. In biopsies which we examined from patients under treatment with this drug (Jamison, Palmer and Vollum 
1961) it was the rapid reduction in the number of cells surrounding the neuro-vascular bundles which was so noticeable.

The radio-active Etisul used in this investigation was generously supplied by the Imperial Chemical Industries Ltd.

\section{References}

Davies, G.E., and Driver, G. W., (1957), "The Anti-tuberculous Activity of Ethyl Thiol-esters, with Particular Reference to Diethyl Dithiolisophthalate", Brit. J. Pharmacol., 12, 434.

DAVEY, T. F., and HogerzeIL, L. M., (1959), "Diethyl Dithiolisophthalate in the Treatment of Leprosy; A Progress Report", Leprosy Rev. 30, 61.

Jamison, D. G., Palmer, E., and Vollum, R. L., (1961), "Preliminary trial with Etisul in Northern Nigeria", Transact. Roy. Soc. Trop. Med. \& Hygiene, 55, 142.

LECHAT, M. F., (1960), "The use of 'Etisul' (Diethyl-Dithiolisophthalate) in the Treatment of Leprosy in Africans", Leprosy Rev., 31, 265.

SNow, G. A., (1957), "The Metabolism of Compounds Related to Ethanethiol", Biochem. J., 65, 77. 